\title{
Controlling 4.0
}

\section{Liebe Leserinnen und Leser,}

vielleicht sind Sie es ja auch langsam leid, ständig irgendeinen Begriff aus der Welt der Wirtschaft mit der ominösen Zahl 4.0 in Verbindung gebracht zu sehen. Zugegeben: wir auch. Dummerweise macht die Digitalisierung aber vor all diesen Begriffen und auch vor dem Controlling nicht halt. Dabei sind zunächst Entwicklungen relevant, die mit Schlagworten wie Self Service, Real-Time-Verarbeitung von Informationen, Standardisierung und Automatisierung, Big Data und Predictive Analytics zu beschreiben sind. Ein prägnantes Beispiel liefert das Forecasting. Einige Pioniere testen derzeit, ob man diesen Prozess nicht völlig automatisieren, das heißt durch die maschinelle Auswertung der vorhandenen Ist-, Plan- und Kontextdaten ersetzen kann. Erste Tests liefern verheißungsvolle Ergebnisse: Die Prognosegüte scheint keineswegs hinter den menschlichen Experten (also den Managern und Controllern) zurückzuliegen - vielleicht auch, weil dem zugrunde liegenden mathematischen Modell politische Spiele gänzlich fremd sind. Im Reporting sind die Pioniere schon weiter, und der aufwendige Prozess ist entweder global im Shared Service zusammengefasst oder in Self-Service-Strukturen weitgehend automatisiert. Aus einer egoistischen Perspektive ist das für die Controller-Zunft eher eine schlechte Entwicklung. Bestätigt sie sich, verlieren Controller zentrale Arbeitsfelder. Digitalisierung wird dann einerseits bedeuten, dass im Controlling signifikante Kapazitäten wegbrechen - warum sollte das im Controlling auch anders sein als anderswo? Andererseits entstehen im Bereich der Analyse potenzielle neue Betätigungsfelder. Hierzu müssen sich Controller aber konsequent und schnell auf die neuen Möglichkeiten einlassen, konkurrieren und kooperieren sie doch in der Regel mit einem neuen Spieler, dem Data Scientist. Wer am Ende die einzige Quelle der Wahrheit im Unternehmen sein wird, ist bei Weitem noch nicht abschließend geklärt.

Die Digitalisierung zwingt schließlich dazu, auch die grundsätzliche Herangehensweise von Controllern infrage zu stellen. Controller sind heute die Hüter und Betreiber der Regelsteuerung. Sie beherrschen den komplexen Prozess souverän, von Forecasts bis hin zu Abweichungsanalysen. Die hohe Unsicherheit einer digitalen Disruption macht das komplexe System allerdings weitgehend obsolet. Komplexität und Dyna-

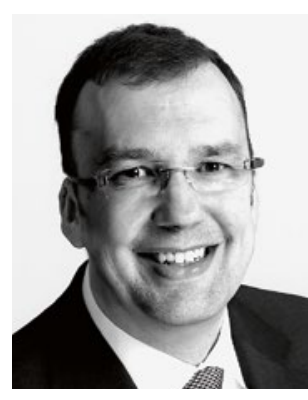

Utz Schäffer



Jürgen Weber mik vertragen sich eben nicht. Management-Prozesse können nicht so weitgehend bürokratisiert werden wie gut planbares Regelgeschäft; die Controlling-Funktionen werden deshalb mehr von den beteiligten Managern selbst ausgeführt werden müssen, weniger von spezialisierten Controllern. Daneben muss ein Portfolio- an die Stelle des Projektdenkens treten, und die Forderung nach „No surprise!“ muss durch eine Kultur des ständigen und zeitnahen Testens und Lernens ersetzt werden. Misserfolge sind dann Lernereignisse auf dem Weg zu einer tragfähigen Lösung.

In der Gesamtschau stellt die Digitalisierung das Controlling also vor signifikante Herausforderungen. Dabei ist sie Chance und Bedrohung zugleich. Noch wird die Herausforderung vielfach negiert, Controlling brauche man doch schließlich immer. Wir sagen: Ja, richtig. Transparenz, Analytik und eine Kultur des gemeinsamen Lernens waren nie so wertvoll wie heute. Aber was gute und vor allem wertstiftende Controller-Praxis ist, wird sich weiter deutlich verändern. Ein schnelles Erkennen des Handlungsdrucks ist daher ebenso erforderlich wie das Bemühen, zeitnah entsprechende Kompetenzen aufzubauen, und dies in instrumenteller und konzeptioneller Hinsicht gleichermaßen.

Sie sehen, es wird wirklich spannend. Viel Spaß bei der Lektüre wünschen Ihnen
